\title{
Intact Visual Perception in Memory-Impaired Patients with Medial Temporal Lobe Lesions
}

\author{
Yael Shrager, ${ }^{1}$ Jeffrey J. Gold, ${ }^{1}$ Ramona 0. Hopkins,${ }^{4,5}$ and Larry R. Squire ${ }^{1,2,3,6}$ \\ Departments of ${ }^{1}$ Neurosciences, ${ }^{2}$ Psychiatry, and ${ }^{3}$ Psychology, University of California, San Diego, La Jolla, California 92093, ${ }^{4}$ Department of Psychology \\ and Neuroscience Center, Brigham Young University, Provo, Utah 84602, ${ }^{5}$ LDS Hospital, Salt Lake City, Utah 84143, and ${ }^{6}$ Veterans Affairs Medical Center, \\ San Diego, California 92161
}

A recent proposal that structures of the medial temporal lobe support visual perception in addition to memory challenges the longstanding idea that the ability to acquire new memories is separable from other cognitive and perceptual functions. In four experiments, we have put this proposal to a rigorous test. Six memory-impaired patients with well characterized lesions of either the hippocampal region or the hippocampal region plus additional medial temporal lobe structures were assessed on difficult tests of visual perceptual discrimination. Across all four experiments, the patients performed as well as controls. The results show that visual perception is intact in memory-impaired patients with damage to the medial temporal lobe even when perception is assessed with challenging tasks. Furthermore, the results support the principle that the ability to acquire new memories is a distinct cerebral function, dissociable from other perceptual and cognitive functions.

Key words: memory; visual perception; anatomy; amnesic; hippocampus; medial temporal lobe

\section{Introduction}

In 1957, Scoville and Milner described the profound effects on memory of bilateral medial temporal lobe resection, which was performed to relieve severe epilepsy in a patient who became known as H.M. This landmark case established that brain structures within the medial temporal lobe are important for memory. Subsequently, animal models of human memory impairment identified the anatomical structures within the medial temporal lobe that are important for understanding H.M.'s memory impairment: the hippocampal region (hippocampus proper, dentate gyrus, and subicular complex) and the perirhinal, entorhinal, and parahippocampal cortices (Squire and Zola-Morgan, 1991; Lavenex and Amaral, 2000). Testing of patient H.M. over the years consistently found intact intellectual and perceptual functions (Milner et al., 1968; Corkin, 1984), suggesting that medial temporal lobe structures are primarily involved in memory. Accordingly, the fundamental idea was advanced that the ability to acquire new memories is a distinct cerebral function, separable from other perceptual and cognitive functions.

This fundamental principle of brain organization has been revisited recently, because there has been interest in the possibility that the structures of the medial temporal lobe might be involved in visual perception in addition to memory. Initially, work focused on the perirhinal cortex. Some experimental studies with

Received Sept. 15, 2005; revised Jan. 12, 2006; accepted Jan. 13, 2006.

This work was supported by the Medical Research Service of the Department of Veterans Affairs, National Institute of Mental Health (NIMH) Grant MH24600, NIMH Training Grant 5-T32-MH20002 (J.J.G.), and a National Defense Science and Engineering Graduate fellowship (Y.S.). We thank Jennifer Frascino and Leah Swalley for assistance.

Correspondence should be addressed to Larry R. Squire, Veterans Affairs Medical Center 116A, 3550 La Jolla Village Drive, San Diego, CA 92161. E-mail: Isquire@ucsd.edu.

DOI:10.1523/JNEUROSCI.4792-05.2006

Copyright $\odot 2006$ Society for Neuroscience $\quad 0270-6474 / 06 / 262235-06 \$ 15.00 / 0$ monkeys underscored the role of perirhinal cortex in memory but found no evidence for a role in visual perception (Buffalo et al., 1999; Hampton and Murray, 2002). In contrast, other studies suggested that the perirhinal cortex might be important for perceptual processing when complex or highly similar visual stimuli are used or stimuli that have a high degree of feature ambiguity (feature overlap) (Buckley and Gaffan, 1998; Murray and Bussey, 1999; Buckley et al., 2001; Bussey and Saksida, 2002; Bussey et al., 2003).

However, it is difficult to test experimental animals for the ability to identify visual stimuli independent of the ability to learn about them, and it has been pointed out that impairments in monkeys that have been attributed to a perceptual deficit could have resulted from impaired learning (Hampton, 2005). In contrast, the distinction between perception and learning is more readily made in studies of humans, because they can be instructed about the requirements of the task. Some studies of patients with large medial temporal lobe lesions, including lesions of the perirhinal cortex, have found intact perceptual abilities (Holdstock et al., 2000; Stark and Squire, 2000; Levy et al., 2005). However, it is of interest that recent assessments of a group of memory-impaired patients, with damage reportedly involving either the hippocampus or the hippocampus plus additional medial temporal lobe structures, found significant impairment on tests of perceptual abilities that involved difficult-to-discriminate faces, objects, and scenes (Lee et al., 2005a,b). This newer work, which involved rather complex visual stimuli, raised the possibility that appropriate tests of memory-impaired patients can reveal perceptual deficits that have not been detected by conventional tests of visual perception (Lee et al., 2005c). Thus, these new findings challenge the long-standing idea that memory impairment can occur as a circumscribed disorder and that memory is 
separable from other cognitive functions. We have reexamined this issue in six patients with damage to the medial temporal lobe who have been thoroughly characterized, both neuropsychologically and neuroanatomically.

\section{Materials and Methods}

Participants. Six memory-impaired patients participated. Four patients (three male) have lesions that are thought to be limited to the hippocampal region (dentate gyrus, CA fields, and subiculum). G.W. (age, 46 years; education, 12 years) and R.S. (age, 49 years; education, 12 years) became amnesic after a drug overdose and associated respiratory failure in 2001 and 1998, respectively. K.E. (age, 64 years; education, 13.5 years) became amnesic in 2004 after an episode of ischemia associated with kidney failure and toxic shock syndrome. L.J. (age, 68 years; education, 12 years) became amnesic in 1988 during a 6 month period with no known precipitating event. Her memory impairment has remained stable since that time. Scores for copy and delayed (12 min) reproduction of the ReyOsterrieth figure (Osterrieth, 1944) (maximum score, 36) were 28.3 and 1.5 , respectively (controls, 30.3 and 20.6). Recall of a short prose passage after a 12 min delay was 0.3 segments for the patients and 6.4 segments for controls (21 segments maximum). Paired-associate learning of 10 noun-noun pairs across three trials was $0,0.5$, and 0.8 pairs for patients and 6.0, 7.6, and 8.9 for controls. Scores for the Wechsler Adult Intelligence Scale-III (WAIS-III) averaged 104 (R.S. took the WAIS-R), and scores for the Delay subscale of the Wechsler Memory Scale-Revised WMS-R averaged 51. Both tests yield means of 100 in the normal population with an SD of 15.

Two patients (both male) have extensive medial temporal lobe lesions as a result of herpes simplex encephalitis (E.P., age of 83 years, education of 12 years, amnesia onset in 1992; G.P., age of 59 years, education of 16 years, amnesia onset in 1987). Copy and delayed scores for the ReyOsterrieth figure were 26.3 and 2.0. Neither patient could recall any of a short prose passage after a 12 min delay ( 0 segments correct), and paired associate learning scores across three trials were 0,0 , and 0 . Scores for the WAIS-III averaged 98, and scores for the Delay subscale of the WMS-R averaged 53 .

Estimates of medial temporal lobe damage were based on quantitative analysis of magnetic resonance (MR) images compared with data for 19 controls (K.E., R.S., and G.W.), 11 controls (L.J.), or four controls (E.P. and G.P.) (Bayley et al., 2005; Gold and Squire, 2005) (for nine coronal MR images from each patient and a control, see supplemental Fig. 1, available at www.jneurosci.org as supplemental material). The volume of the full anteroposterior length of the hippocampus and the parahippocampal gyrus were measured using criteria based on histological analysis of healthy brains (Amaral and Insausti, 1990; Insausti et al., 1998a,b). For each patient, the hippocampal and parahippocampal gyrus volumes were divided by the intracranial volume (normalized) to correct for brain size (Gold and Squire, 2005). K.E., L.J., R.S., and G.W. have an average bilateral reduction in hippocampal volume of $49,46,33$, and $48 \%$, respectively (all values $>3.0$ SDs below the control mean). In comparison, the volume of the parahippocampal gyrus (temporopolar cortex and perirhinal, entorhinal, and parahippocampal cortices) is reduced by 17 , $-8,1$, and $12 \%$, respectively (all values within 2 SDs of the control mean). E.P. and G.P. have an average bilateral reduction in hippocampal volume of 97 and 96\%, respectively. In addition, E.P. and G.P. have an average bilateral reduction in the volume of the parahippocampal gyrus of 93 and $92 \%$, respectively, reflecting a complete loss of temporopolar, perirhinal, and entorhinal cortices bilaterally and significant damage to parahippocampal cortex (Bayley et al., 2005).

Additional measurements, based on four controls for each patient, were performed for the insular cortex, fusiform gyrus, frontal lobes, lateral temporal lobes, parietal lobes, and occipital lobes. The only volume reductions in these regions $>1.3$ SDs of the control mean were the parietal lobe for R.S. (Bayley et al., 2005), the fusiform gyrus of E.P. and G.P. (54 and $48 \%$ reduced, respectively), and the insular cortex of G.P. (65\% reduced).

Eight healthy controls (all male; mean age, 70.8 years; range, 58-84 years; mean education, 13.4 years) participated in the behavioral experiments.
Stimuli. The test stimuli were morphed grayscale images in each of three stimulus categories: faces, objects, and scenes. Morphed images were created by gradually morphing one distinct grayscale image into another (e.g., one hat into a different hat or a lemon into a tennis ball) across a 100 step series using computer software (Morpheus Photo Animator; ACD Systems, Saanichton, British Columbia, Canada). One distinct image was labeled 01, the other distinct image was labeled 100, and the intermediate images (02-99) were morphs that progressed from image 01 to image 100 .

Experiment 1: visual discrimination learning. Experiment 1 was designed to duplicate the conditions of task 1 of Lee et al. (2005a). Four different tests in each of three stimulus categories (53 trials per test, 12 tests in total). All of the images in each test were derived from the same pair of distinct images. On trials 1-3, two distinct images were presented on the screen (see Fig. 1a). One of the images was arbitrarily designated as "correct," and participants were asked to indicate on each trial which image they believed to be the correct one. Trials were self-paced, and feedback was provided after each response (a high tone for correct and a low tone for incorrect). On trials $4-53$, two morphed images were presented, and participants tried to identify which morphed image was more similar to the distinct image that was designated as correct on trials 1-3. Trials $4-53$ were given at five different levels of difficulty (1-5). Thus, trials for level 1 (the easiest level) used images 01-10 and images 91-100 to create 10 image pairs (e.g., image 01 paired with 100 , image 02 paired with 99 , and so on). Trials for level 5 (the most difficult level) used images 41-50 and images 51-60 to create 10 additional image pairs (e.g., image 41 paired with image 60 , image 42 paired with image 59 , and so on). The same procedure was followed to create the image pairs for levels 2-4. Two trials from each level of difficulty were presented every 10 trials. The 12 different tests were presented in four different orders across participants, with the constraint that the same stimulus category was never tested more than twice in succession.

Experiment 2: visual discrimination. Experiment 2 was designed to duplicate the conditions of task 2 of Lee et al. (2005a). Four different tests were given in each of three stimulus categories ( 50 trials per test, 12 tests in total). The images in each test were all derived from the same pair of distinct images. On each trial, a pair of morphed images was presented on the screen below one of the distinct images from which that pair was derived (see Fig. 2a). Participants were asked to indicate which of the two morphed images was more similar to the distinct image. Trials were given at five different levels of difficulty (1-5; for details on construction of image pairs at each level, see experiment 1). Within each test, two trials from each level of difficulty were presented every 10 trials. Trials were self-paced, and feedback was provided on each trial. The 12 different tests were presented in four different orders across participants, with the constraint that the same stimulus category was never tested more than twice consecutively.

Experiment 3: trial-unique visual discrimination. Experiment 3 consisted of 40 unique trials in each of three stimulus categories (three tests in total). On each trial, a pair of morphed images was presented on the screen below one of the distinct images from which that pair was derived (see Fig. $3 a$ ). The morphed images for each trial were always derived from a unique pair of distinct images. Participants were asked to indicate which of the two morphed images was more similar to the distinct image. For each stimulus category, 20 trials were given at each of two levels of difficulty (levels 4 and 5; for details on construction of image pairs at each level, see experiment 1). Five trials from each level of difficulty were presented every 10 trials. Trials were self-paced, and feedback was provided on each trial. The three different tests were presented in four different orders across participants.

Experiment 4: visual matching. Experiment 4 consisted of 45 unique trials ( 15 trials in each of three stimulus categories). On each trial, a target image derived from a unique pair of distinct images was presented at the top of the screen (see Fig. $4 a$ ). This image was randomly selected from images $21-80$ in the 100-image series. In addition, a single image chosen randomly from the 100-image series was presented below the target image. Participants were asked to match the lower image to the target image by scrolling through the ordered series of 100 morphed images, viewing only one image at a time, and selecting the image that was identical to the 

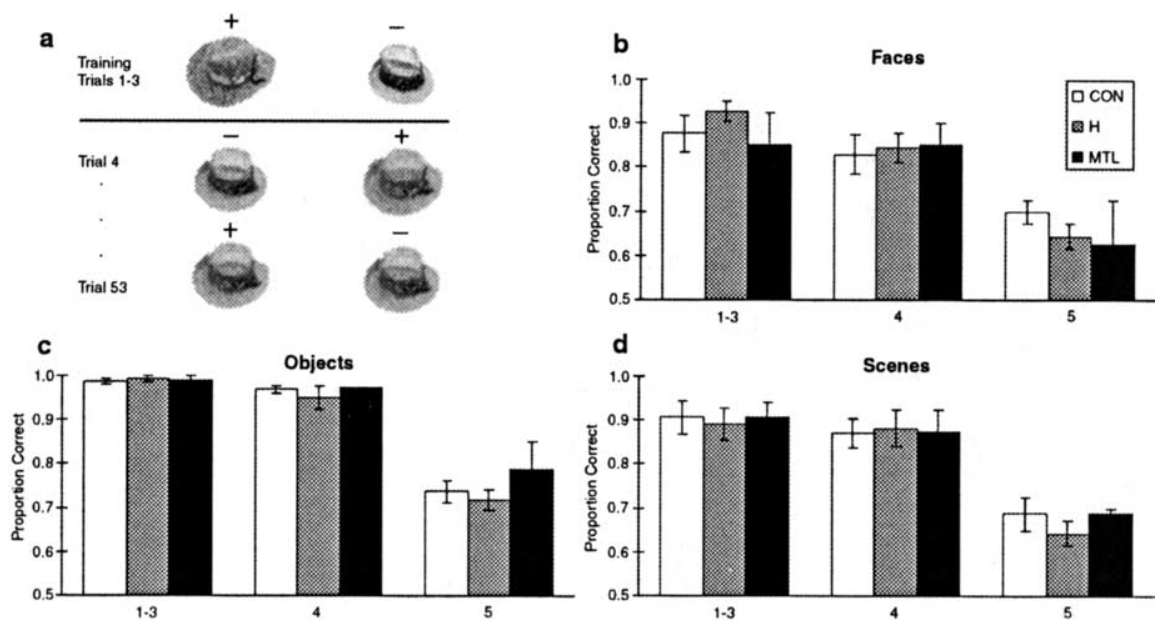

Figure 1. Visual discrimination learning. $\boldsymbol{a}$, The task. On trials 1-3, two distinct images were presented. Participants were asked to indicate which image they believed to be "correct" (here identified by a + ), and feedback was provided after each choice. For trials $4-53$, participants saw two morphed images, each of which was intermediate to the images in trials $1-3$. On each trial, participants chose the image that appeared more similar to the correct image. $\boldsymbol{b}-\boldsymbol{d}$, Proportion correct scores for the $\mathrm{H} \mathrm{group}(n=$ 4), the MTL group $(n=2)$, and the CON group ( $(n=8)$ on four different tests in each of three categories: $\boldsymbol{b}$, faces; $\boldsymbol{c}$, objects; $\boldsymbol{d}$, scenes. Trials were given at five different levels of difficulty (1-5), and scores are shown for levels $1-3$, level 4 , and level 5 . Error bars indicate SEM.

a
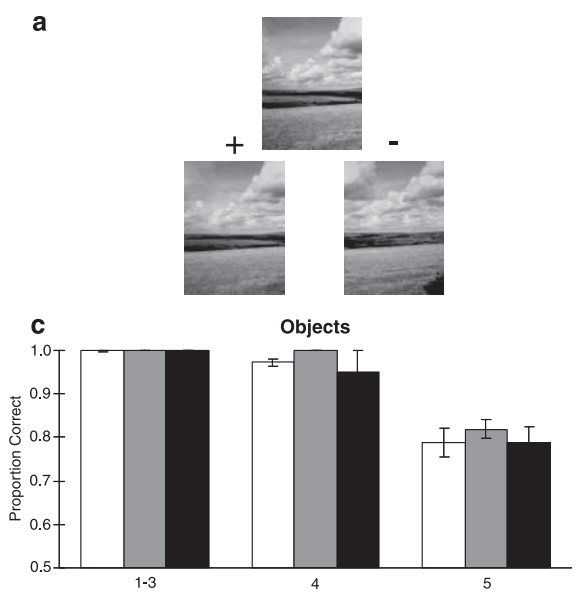
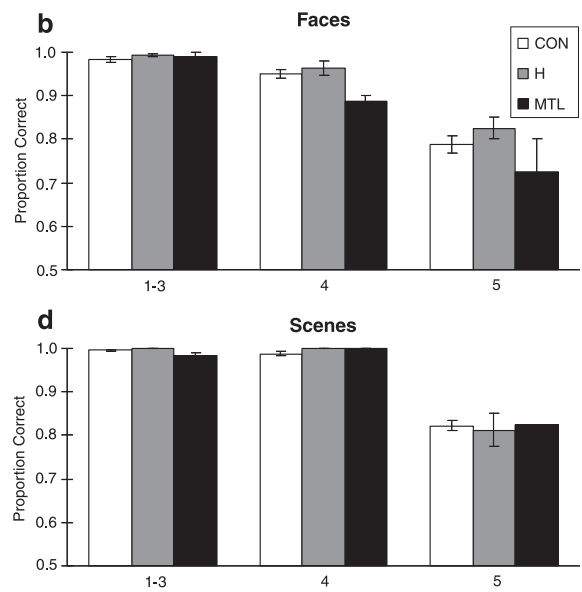

Figure 2. Visual discrimination. $\boldsymbol{a}$, The task. On each trial, two morphed images were presented below a single distinct image. Participants were asked to choose the lower image (here identified by a + ) that appeared more similar to the upper image. $\boldsymbol{b}-\boldsymbol{d}$, Proportion correct scores for the H group $(n=4)$, the MTL group $(n=2)$, and the CON group $(n=8)$ on four different tests in each of three categories: $\boldsymbol{b}$, faces; $\boldsymbol{c}$, objects; $\boldsymbol{d}$, scenes ( 50 trials per test). The morphed images presented in each test of 50 trials were all derived from the same two source images (see Materials and Methods). Trials were given at five different levels of difficulty (1-5), and scores are shown for levels 1-3, level 4, and level 5 . Error bars indicate SEM.

target. Trials were self-paced, and feedback was not provided. The 45 trials were presented in four different orders across participants, with the constraint that the same stimulus category was never tested more than three times in succession.

\section{Results}

Experiment 1: visual discrimination learning

Figure $1 b-d$ shows scores for controls (CON), patients with hippocampal lesions $(\mathrm{H})$, and patients with large lesions of the medial temporal lobe (MTL) on tests of visual discrimination learning involving faces, objects, and scenes. An ANOVA (stimulus category $\times$ group $)$ revealed an effect of category $\left(F_{(2,22)}=7.9\right.$; $p=0.003)$ but no effect of group $(p=0.993)$ and no interaction $(p=0.88)$. A second ANOVA that included difficulty level (stimulus category $\times$ difficulty level $\times$ group) also found no effect of group $(p=0.96)$ and no interactions (all $p$ values $>0.2$ ). Indeed, none of the 18 possible pairwise comparisons between the patients and controls approached significance (all $t$ values $<1.3$; all $p$ values $>0.22$ ). Furthermore, the mean scores of the patient groups were numerically higher than the corresponding score of the control group in 9 of the 18 cases. Despite the normal performance of the patients overall, two scores merit separate mention. In difficulty levels 1-3 and 5 of the faces test, patient E.P. in the MTL group obtained low scores ( 0.78 and 0.53 , respectively), because he had difficulty remembering across the 50 trials which face was correct. On difficulty levels $1-3$, the range of scores for the $\mathrm{CON}$ group was $0.65-1.00$, and, on difficulty level 5, the range of CON scores was $0.58-$ 0.80. An ANOVA across the five 10-trial blocks within each test revealed a linear contrast of block $(p<0.001)$ but no effect of group and no interaction ( $p$ values $>0.1)$. The linear contrast was found for both controls and patients separately ( $p$ values $<0.06$ )

\section{Experiment 2: visual discrimination}

Figure $2 b-d$ shows scores for the three groups (CON, H, and MTL) on tests of visual discrimination involving faces, objects, and scenes. An ANOVA (stimulus category $\times$ group) revealed an effect of category $\left(F_{(2,22)}=6.0 ; p=0.008\right)$ but no effect of group ( $p=0.11)$ and no interaction $(p=0.73)$. A second ANOVA that included difficulty level (stimulus category $\times$ difficulty level $\times$ group) also found no effect of group ( $p=0.12$ ) and no interactions involving the group factor (all $p$ values $>0.4$ ). Furthermore, of 18 possible pairwise comparisons between the patients and controls, the mean scores of the patient groups were numerically better than the corresponding score of the control group in 12 cases. There was only one instance in which a patient group performed more poorly than the controls, although the mean difference between groups was small ( 0.89 vs 0.95 ; MTL group vs CON group; level 4 of faces, $\left.t_{(8)}=3.24 ; p=0.01\right)$. In that condition, the range of scores for the CON group was 0.93-1.0. Another condition that deserves mention is difficulty level 5 of the faces test, in which E.P. obtained a low score (0.65). On that test, the range of scores for the CON group was $0.68-0.85$. An ANOVA across the five 10trial blocks within each test (block $\times$ group) revealed no linear contrast of block, no effect of group, and no interaction ( $p$ values $>0.1)$.

\section{Experiment 3: trial-unique visual discrimination}

Figure $3 b-d$ shows scores for the three groups $(\mathrm{CON}, \mathrm{H}$, and MTL) on trial-unique tests of visual discrimination involving faces, objects, and scenes. An ANOVA (stimulus category $\times$ difficulty level $\times$ group) revealed effects of category $\left(F_{(2,22)}=8.8\right.$; 
$p=0.002)$ and difficulty level $\left(F_{(1,11)}=\right.$ $61.2 ; p<0.001)$ but no effect of group $(p=0.99)$ and no interactions (all $p$ values $>0.7$ ). Of 12 possible pairwise comparisons between the patients and controls, none approached significance (all $t$ values $<1.06$; all $p$ values $>0.3$ ). In addition, the mean scores of the patient groups were numerically better than the corresponding score of the control group in 8 of the 12 cases. Note that level 5 of the faces test was so difficult that none of the groups scored significantly above chance. When the data analysis was based only on level 4, there was still no effect of group $(p=0.92)$, and the two patient groups both scored numerically above the control mean.

\section{Experiment 4: visual matching}

Figure $4 b$ shows scores for the three groups (CON, H, and MTL) on a test of visual matching involving faces, objects, and scenes. An ANOVA (stimulus category $\times$ group) revealed an effect of category $\left(F_{(2,22)}=12.5 ; p<0.001\right)$ but no effect of group $(p=0.41)$ and no interaction $(p=0.83)$. The patient groups scored numerically better (lower) than the control group in all six possible comparisons.

\section{Discussion}

Two groups of memory-impaired patients (four with hippocampal lesions and two with large medial temporal lobe lesions) were given four difficult tests of visual perceptual discrimination. With one exception, the two groups performed as well as controls. The exception occurred in difficulty level 4 of the faces test in experiment 2, in which the two patients with large medial temporal lobe lesions performed a little more poorly than controls (E.P., 0.90; G.P., 0.88; CON, 0.95). Additionally, in three other instances (the faces test of experiment 1 , difficulty levels $1-3$ and 5; the faces test of experiment 2, difficulty level 5), patient E.P. obtained scores near the bottom of the control range.

It seems likely that the slightly lower scores in the four justmentioned conditions were attributable to severe memory impairment. In experiment 1, participants had to learn across trials which image was correct. E.P. in particular indicated on a few occasions that he had difficulty remembering the correct image. In experiment 2, groups of consecutive trials were derived from the same two faces, and participants with intact memory could potentially benefit from what they had encountered on previous trials. Both patients with large lesions, especially E.P., had to be instructed repeatedly about the task requirements and could have had difficulty retaining information about the stimuli from trial to trial.

It is also possible that E.P.'s somewhat lower performance in experiments 1 and 2 resulted from a specific difficulty with faces. In previous studies, E.P. performed numerically, albeit not significantly, worse than controls on tasks involving complex face stimuli (Stark and Squire, 2000; Levy et al., 2005). Also, E.P. and G.P. were mildly impaired at perceiving certain facial emotions, perhaps because of damage to the amygdala or fusiform gyrus (Schmolck and Squire, 2001). Still, it is interesting that E.P. and G.P. were intact on the faces tests of experiments 3 and 4 despite their damage to the fusiform gyrus. Indeed, all of the patients
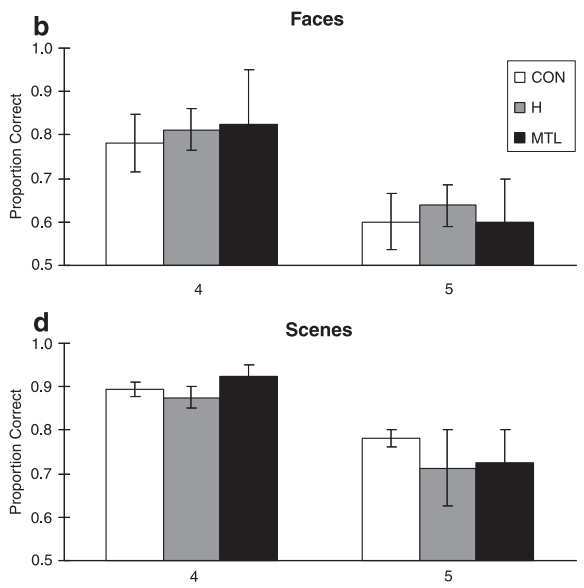

a

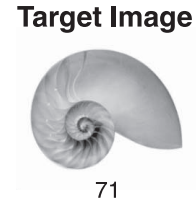

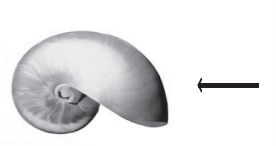

01

b

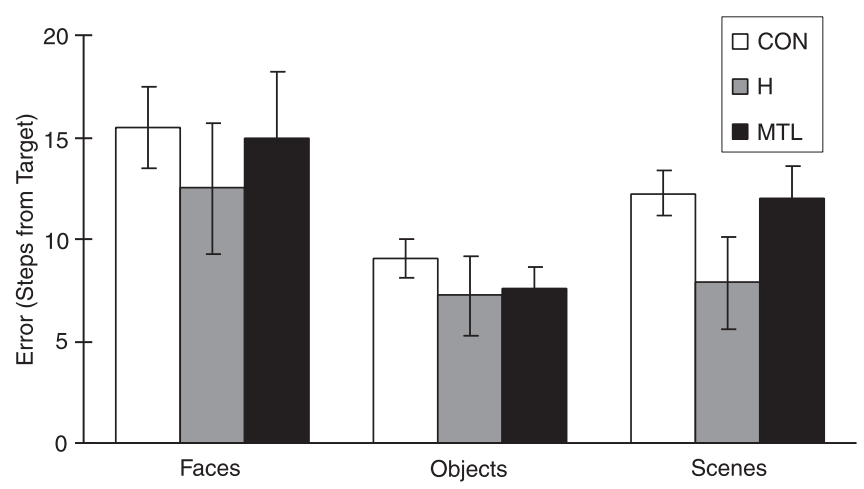

Figure 4. Visual matching. $\boldsymbol{a}$, The task. On each of 45 unique trials, a target image was presented above a single image. Both images were derived from a unique pair of distinct images (images 01 and 100). In the case illustrated, the target image is image number 71 in the 01-100 series, and the bottom image is image number 64 from the same series. Participants were asked to scroll through the ordered series of 100 images (images $01-100$ ) and to try to match the lower image to the target. Image numbers did not appear during testing. $\boldsymbol{b}$, Error scores (number of steps from target) for the Hroup $(n=4)$, the MTL group $(n=2)$, and the CON group $(n=8)$ on trials involving faces, objects, and scenes ( 15 trials per category). Error bars indicate SEM.

performed as well as controls in the faces tests reported in experiments 3 and 4, in which all of the trials were unique and in which learning could not have contributed to performance. It therefore seems most likely that the occasional low performance in experiments 1 and 2 resulted from impaired learning and memory. 
The present findings agree with a number of other reports of intact visual perception after damage to the medial temporal lobe. Thus, monkeys with lesions of the perirhinal cortex were slow to learn visual discriminations but then performed as well as controls on transfer trials in which the original stimuli were rotated, enlarged, shrunk, presented with color removed, or degraded with masks (Hampton and Murray, 2002). Furthermore, memory-impaired patients with medial temporal lobe lesions also exhibited intact perception, as measured by tests involving complex, abstract designs (Holdstock et al., 2000) and complex, highly similar images (Stark and Squire, 2000; Levy et al., 2005).

In other work, memory-impaired patients with damage reportedly involving the medial temporal lobe (including perirhinal cortex) were impaired on visual perceptual tests of difficultto-discriminate faces, objects, and scenes (Lee et al., 2005a,b). A second group of patients with damage reportedly involving the hippocampus was impaired on visual perceptual tests of scenes (Lee et al., 2005a,b). Our experiments 1 and 2 attempted to duplicate the conditions of the first of these studies (Lee et al., 2005a). Insofar as was possible, we used identical stimuli and tasks, and the difficulty of our tasks was virtually identical to the difficulty of the tasks used by Lee et al. (2005a). Thus, control performance in our experiment 2 was 0.99 for levels $1-3,0.97$ for level 4 , and 0.80 for level 5 . In the corresponding experiment by Lee et al. (2005a), the only experiment in which control scores were provided, we estimated from their Figure 4 that the control scores were $1.0,0.98$, and 0.81 for levels $1-3,4$, and 5 , respectively.

Across experiments 1 and 2, our two patients with large medial temporal lobe lesions obtained mean $z$-scores of -0.60 , +0.42 , and -0.26 for faces, objects, and scenes, respectively, and our four patients with hippocampal lesions obtained mean $z$-scores of $+0.5,+0.15$, and +0.11 . As estimated from Figure 3 by Lee et al. (2005a), the $z$-scores across the two corresponding experiments for three patients with medial temporal lobe lesions averaged $-4.8,-2.2$, and -7.3 for faces, objects, and scenes, respectively, and the $z$-scores for four patients with hippocampal lesions averaged $0,-0.13$, and -2.1 . Notably, in the four cases in which Lee et al. (2005a) reported an overall impairment (MTL group: faces, objects, scenes; H group: scenes), the $z$-scores obtained by their patients were more than 2 SDs lower than the $z$-scores obtained by our patients.

In two additional experiments, we used trial-unique stimuli to eliminate entirely any possible contribution of learning to performance of the perceptual tasks. Again, the patients performed as well as controls (MTL group, mean $z$-score of +0.08 ; $\mathrm{H}$ group, mean $z$-score of +0.31 ). Given that we can identify no substantive differences between the materials and procedures that we used and those used by Lee et al. (2005a), we looked for possible differences in the two patient groups to understand the discrepancy in findings.

The lesions in the patients studied by Lee et al. (2005a,b) were characterized by visual ratings of magnetic resonance images (four- or five-point scales). Ratings based on visual inspection, however, are not the same as quantitative measurements of brain tissue. Additionally, the ratings given for each patient were based on a single coronal section from the anterior hippocampus, posterior hippocampus, amygdala, and lateral temporal lobe and four coronal sections from the medial temporal lobe cortices (one each from entorhinal cortex, transentorhinal cortex, perirhinal cortex, and the medial bank of the occipitotemporal sulcus). Using single sections to assess damage in these structures leaves a considerable amount of tissue unexamined (see below). Furthermore, even by these incomplete assessments, the damage in some patients extended beyond the brain structures that defined the groups. Indeed, two of the four patients in the hippocampal group had significant $(>2$ SDs from the control mean) damage to the parahippocampal gyrus, one of these two had significant damage to the anterior temporal lobe, and one of the three patients in the medial temporal lobe group had significant damage to the lateral temporal lobe. In the absence of thorough, quantitative assessment of lesions, the possibility remains that there is additional damage outside of the hippocampus (in the case of the hippocampal group) or the medial temporal lobe (in the case of the medial temporal lobe group).

The lesions of the patients in the present study were rigorously measured using quantitative volumetric analysis of magnetic resonance images (Bayley et al., 2005; Gold and Squire, 2005), using criteria based on histological analyses of healthy brains (Amaral and Insausti, 1990; Insausti et al., 1998a,b). For each patient, $\sim 60$ sections were measured in $1 \mathrm{~mm}$ intervals rostrocaudally through the medial and lateral temporal lobes. Measurements were taken in every section in which the structure of interest was present, not just in a single section (mean of 29 sections for the hippocampal region, 15 for the temporopolar cortex, 30 for the perirhinal cortex, 24 for the entorhinal cortex, 16 for the parahippocampal cortex, and 58 for the lateral temporal lobe). In addition, volumes were calculated for the insular cortex, fusiform gyrus, and the frontal, parietal, and occipital lobes.

Studies with monkeys have reported that damage to the perirhinal cortex impaired performance on difficult perceptual tasks involving complex and highly similar stimuli (Buckley and Gaffan, 1998; Buckley et al., 2001; Bussey and Saksida, 2002; Bussey et al., 2003). As discussed previously (Levy et al., 2005; Squire et al., 2006), it is difficult with experimental animals to distinguish between an impaired perceptual ability to identify objects and an impaired ability to learn about those objects. Indeed, a recent review of this literature concluded that impairments in animals that have been attributed to a perceptual deficit likely resulted from impaired learning (Hampton, 2005) [for responses, see Buckley (2005) and Bussey et al. (2005)].

In a previous study (Levy et al., 2005), patient E.P. performed normally on a difficult visual perceptual discrimination task involving the blending of two unrelated images when learning was not required (experiment $2 \mathrm{a}$ ) but then had marked difficulty when an explicit learning requirement was introduced (experiment 3). Interestingly, in experiment 1 of the current study (in which there was an explicit learning requirement), E.P.'s learning problem appeared to impact his performance only a little on the faces test and not at all on the objects and scenes tests. There are a number of differences between the tests of blended stimuli given previously (Levy et al., 2005) and the tests of morphed stimuli given in the current experiment 1 , including the use of practice trials with easy-to-discriminate stimuli in the current experiment. E.P. was apparently better able to label and rehearse the stimuli in the current experiment 1 than in the previous study (Levy et al., 2005).

In experiments 3 and 4 , we isolated the process of visual perceptual discrimination by removing the need to learn the correct responses (as could have occurred in experiment 1) and by using tests in which the stimuli on every trial were derived from a unique pair of images to remove any possible contribution of learning and memory (which could have been a factor in experiment 2). In these cases, the patients could not be disadvantaged as a result of their poor memory for previous trials. The tasks were perceptually demanding, as indicated by the fact that controls consistently achieved less than maximum scores in the most dif- 
ficult conditions of each experiment. In all of the conditions of experiments 3 and 4, the patients performed as well as controls.

In summary, visual discrimination performance was intact despite damage to the hippocampal region or damage to the medial temporal lobe that included the hippocampal region and the perirhinal cortex. Over the past 40 years, numerous studies of memory-impaired patients with lesions of the medial temporal lobe have found visual perceptual function to be intact (Milner et al., 1968; Corkin, 1984; Stark and Squire, 2000; Levy et al., 2005). It was this early work that led to the principle that memory can be severely impaired without impairing other intellectual or perceptual functions. By using more difficult test material than has been used previously and by testing patients with thoroughly characterized lesions, our study put this principle to a particularly rigorous test. We found that visual perception is intact despite extensive medial temporal lobe damage, even when perception is challenged with difficult tasks involving complex and highly similar images.

\section{References}

Amaral DG, Insausti R (1990) The human hippocampal formation. In: The human nervous system, pp 711-755. San Diego: Academic.

Bayley PJ, Gold JJ, Hopkins RO, Squire LR (2005) The neuroanatomy of remote memory. Neuron 46:799-810.

Buckley MJ (2005) The role of the perirhinal cortex and hippocampus in learning, memory, and perception. Q J Exp Psychol B 58:246-268.

Buckley MJ, Gaffan D (1998) Perirhinal cortex ablation impairs visual object identification. J Neurosci 18:2268-2275.

Buckley MJ, Booth MC, Rolls ET, Gaffan D (2001) Selective perceptual impairments after perirhinal cortex ablation. J Neurosci 21:9824-9836.

Buffalo EA, Ramus SJ, Clark RE, Teng E, Squire LR, Zola SM (1999) Dissociation between the effects of damage to perirhinal cortex and area TE. Learn Mem 6:572-599.

Bussey TJ, Saksida LM (2002) The organization of visual object representations: a connectionist model of effects of lesions in perirhinal cortex. Eur J Neurosci 15:355-364.

Bussey TJ, Saksida LM, Murray EA (2003) Impairments in visual discrimination after perirhinal cortex lesions: testing "declarative" vs. "perceptual-mnemonic" views of perirhinal cortex function. Eur J Neurosci 17:649-660.

Bussey TJ, Saksida LM, Murray EA (2005) The perceptual-mnemonic/feature conjunction model of perirhinal cortex function. Q J Exp Psychol B 58:269-282.

Corkin S (1984) Lasting consequences of bilateral medial temporal lobectomy: clinical course and experimental findings in H.M. Semin Neurol 4:249-258.

Gold JJ, Squire LR (2005) Quantifying medial temporal lobe damage in memory-impaired patients. Hippocampus 15:79-85.
Hampton RR (2005) Monkey perirhinal cortex is critical for visual memory, but not for visual perception: re-examination of the behavioural evidence from monkeys. Q J Exp Psychol B 58:283-299.

Hampton RR, Murray EA (2002) Learning of discriminations is impaired, but generalization to altered views is intact, in monkeys (Macaca mulatta) with perirhinal cortex removal. Behav Neurosci 116:363-377.

Holdstock JS, Gutnikov SA, Gaffan D, Mayes AR (2000) Perceptual and mnemonic matching-to-sample in humans: contributions of the hippocampus, perirhinal and other medial temporal lobe cortices. Cortex 36:301-322.

Insausti R, Insausti AM, Sobreviela MT, Salinas A, Martinez-Penuela JM (1998a) Human medial temporal lobe in aging: anatomical basis of memory preservation. Microsc Res Tech 43:8-15.

Insausti R, Juottonen K, Soininen $\mathrm{H}$, Insausti AM, Partanen K, Vainio P, Laakso MP, Pitkanen A (1998b) MR volumetric analysis of the human entorhinal, perirhinal, and temporopolar cortices. AJNR Am J Neuroradiol 19:659-671.

Lavenex P, Amaral DG (2000) Hippocampal-neocortical interaction: a hierarchy of associativity. Hippocampus 10:420-430.

Lee AC, Bussey TJ, Murray EA, Saksida LM, Epstein RA, Kapur N, Hodges JR, Graham KS (2005a) Perceptual deficits in amnesia: challenging the medial temporal lobe "mnemonic" view. Neuropsychologia 43:1-11.

Lee AC, Buckley MJ, Pegman SJ, Spiers H, Scahill VL, Gaffan D, Bussey TJ, Davies RR, Kapur N, Hodges JR, Graham KS (2005b) Specialization in the medial temporal lobe for processing of objects and scenes. Hippocampus 15:782-797.

Lee AC, Barense MD, Graham KS (2005c) The contribution of the human medial temporal lobe to perception: bridging the gap between animal and human studies. Q J Exp Psychol B 58:300-325.

Levy DA, Shrager Y, Squire LR (2005) Intact visual discrimination of complex and feature-ambiguous stimuli in the absence of perirhinal cortex. Learn Mem 12:61-66.

Milner B, Corkin S, Teuber H-L (1968) Further analysis of the hippocampal amnesic syndrome: 14-year follow-up study of H.M. Neuropsychologica 6:215-234.

Murray EA, Bussey TJ (1999) Perceptual-mnemonic functions of the perirhinal cortex. Trends Cogn Sci 3:142-151.

Osterrieth PA (1944) Le test de copie d'une figure complexe [The test of copying a complex figure]. Arch Psychol 30:206-356.

Schmolck H, Squire LR (2001) Impaired perception of facial emotions following bilateral damage to the anterior temporal lobe. Neuropsychology 15:30-38

Scoville WB, Milner B (1957) Loss of recent memory after bilateral hippocampal lesions. J Neurochem 20:11-21.

Squire LR, Zola-Morgan S (1991) The medial temporal lobe memory system. Science 253:1380-1386.

Squire LR, Shrager Y, Levy DA (2006) Lack of evidence for a role of medial temporal lobe structures in visual perception. Learn Mem, in press.

Stark CE, Squire LR (2000) Intact visual perceptual discrimination in humans in the absence of perirhinal cortex. Learn Mem 7:273-278. 